\title{
Nanoscale Stiffness Distribution in Bone Metastasis
}

\author{
Ludovic Richert ${ }^{*}$, Laetitia Keller ${ }^{2,3 *}$, Quentin Wagner ${ }^{2,3}$, Fabien Bornert ${ }^{2,3,4}$, \\ Catherine Gros 2,3,4, Sophie Bahi2,3,4, François Clauss',3,4, William Bacon 2,3,4, \\ Philippe Clézardin5 ${ }^{5}$ Nadia Benkirane-Jessel $2,3,4$, Florence Fioretti $2,3,4$
}

${ }^{1}$ Centre National de la Recherche Scientifique (CNRS), UMR, Faculté de Pharmacie de I'Université de Strasbourg (UdS), Illkirch, France

${ }^{2}$ Institut National de la Santé et de la Recherche Médicale (INSERM), Osteoarticular and Dental Regenerative Nanomedicine, UMR, Faculté de Médecine de I'Université de Strasbourg and FMTS, Strasbourg, France

${ }^{3}$ Faculté de Chirurgie Dentaire de l'Université de Strasbourg (UdS), Strasbourg, France

${ }^{4}$ Hôpitaux Universitaires de Strasbourg, Strasbourg, France

${ }^{5}$ Institut National de la Santé et de la Recherche Médicale (INSERM), UMR, Faculté de Médecine Laënnec de I’Université Claude Bernard Lyon 1, Lyon, France

Email: f.fioretti@unistra.fr

Received 21 August 2015; accepted 28 December 2015; published 31 December 2015

Copyright (C) 2015 by authors and Scientific Research Publishing Inc.

This work is licensed under the Creative Commons Attribution International License (CC BY). http://creativecommons.org/licenses/by/4.0/

(c)

\begin{abstract}
Nanomechanical heterogeneity is expected to have an effect on elasticity, injury and bone remodelling. In normal bone, we have two types of cells (osteoclasts and osteoblasts) working together to maintain existing bone. Bone cancers can produce factors that make the osteoclasts work harder. This means that more bone is destroyed than rebuilt, and leads to weakening of the affected bone. We report here the first demonstration of the nanoscale stiffness distribution in bone metastases before and after treatment of animals with the bisphosphonate Risedronate, a drug which is currently used for the treatment of bone metastases in patients with advanced cancers. The strategy used here is applicable to a wide class of biological tissues and may serve as a new reflection for biologically inspired scaffolds technologies.
\end{abstract}

\section{Keywords}

Bone Metastasis, Stiffness, Risedronate

\section{Introduction}

As cancer becomes more advanced, it tends to spread throughout the body, with the bones being a common site

*These authors contributed equally to this work.

How to cite this paper: Richert, L., Keller, L., Wagner, Q., Bornert, F., Gros, C., Bahi, S., Clauss, F., Bacon, W., Clézardin, P., Benkirane-Jessel, N. and Fioretti, F. (2015) Nanoscale Stiffness Distribution in Bone Metastasis. World Journal of Nano Science and Engineering, 5, 219-228. http://dx.doi.org/10.4236/wjnse.2015.54023 
of spread for many cancers [1]. Spread of cancer to the bone from its original site is referred to as bone metastases. Treatment may consist of radiation therapy, bisphosphonates, hormone therapy and/or chemotherapy, depending upon the type of cancer from which the metastasis originated [2] [3].

Researchers are evaluating ways to avoid or decrease the ache or break caused by bone metastasis, not just provide treatment once they occur. Myeloma and some secondary bone cancers can produce factors that make the osteoclasts work harder [1]-[4]. This means that more bone is destroyed than rebuilt, and leads to weakening of the affected bone. This can cause pain and means that the bone can fracture or break more easily; in this case, ablation of metastasis bone and implantation of biomaterials are needed.

As many natural materials, bone is heterogeneous. Mechanical heterogeneity is expected to exist at different length scales. It has become evident that the nanoscale properties of bone participate in its macroscopic biomechanical funcion [4]-[7]. At the macroscopic level, considerable variations in mechanical properties have been detected for different tissues locations [8], as well as for regions within a specific location [9]. Microscopically, indentation has further identified differences in modulus and hardness for specific features such as lamellae (thin and thick) in osteons of bone which have been recognized as collagen fibril orientation, as well as variations in mineral composition [10] [11]. The cellular remodelling process resulting in a combination of new and old bone is a result of this heterogeneity at this length scale [12]. Atomic force microscopy (AFM)-based nanoindentation has been used to distinguish mechanically heterogeneous microscale regions in bone tissue from genetically modified mice [13]-[16]. These studies also raise important issues as to whether heterogeneity is advantageous or disadvantageous to the mechanical function of bone [17]. Recently, the detailed study of the consequences of heterogeneity, in particular at the nanoscale, was reported [18].

An inherent feature of bones is their heterogeneity in the collagen fibril orientation and the mineral content. This particular spatial structure has a direct incidence on their elasticity even on the nanometer scale [18]. The elasticity, or equivalently the stiffness, of a material can be quantified using nano-indentation. It consists in fixing a probe (most often a sphere, a cone or a pyramid) at the end of the cantilever of an atomic force microscope (AFM) and to measure the deflection of the cantilever when the material to be characterized is pushed into contact with the probe. The applied force is derived from the deflection, and the indentation is derived from the deflection and the material displacement. In this way, we obtain a so-called force curve. If the material behaves as an elastic solid, a force curve can be interpreted using Hertzian mechanics [19]. This characterization method was used to compare healthy bone, bone with metastases, and bone after treatment with bisphosphonates.

Bisphosphonates drugs are used for the treatment of cancer-related hypercalcemia and treatment of bone metastases in patients with advanced cancers [2]. Bisphosphonates decrease the rate of bone destruction in patients with bone metastases and clinical studies have demonstrated that bisphosphonates can significantly decrease the pain and number of fractures induced by bone metastases.

Bisphosphonates are established in the treatment of skeletal metastases [12]. They have a common P-C-P structural feature consisting in a central carbon atom correlated to two phosphonate moieties, and two substituents (R1, R2) (Figure 1). Phosphonate and R1 (preferably hydroxyl) groups allow bisphosphonates to bind<smiles>[R]C([R])(P(=O)(O)O)P(=O)(O)O</smiles>

(a)

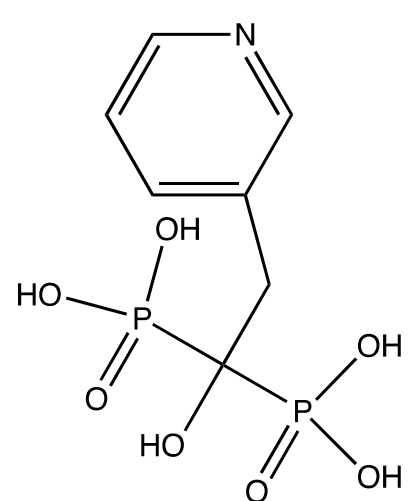

(b)

Figure 1. (a) Chemical structure of bisphosphonate drugs. P-C-P common structural feature of bisphosphonates and location of the specific R1 and R2; (b) Chemical structure of the Risedronate (RIS) antitumor drug (2-(3-pyridinyl)-1-hydroxyethylidenebisphosphonic acid). 
avidly to bone hydroxyapatite, while R2 determines their effectiveness to inhibit osteoclast-mediated bone resorption [3]. Clinically, bisphosphonates significantly diminish bone destruction rate, pain and fractures associated with bone metastases [2]. Moreover, there is extensive in vivo preclinical evidence that bisphosphonates reduce skeletal tumor burden and inhibit bone metastasis formation in animals [12]. Among proposed mechanisms of actions, bisphosphonates may render bone a less favorable microenvironment for metastasis development by reducing osteoclast-mediated bone resorption, which, in turn, deprives tumor cells of bone-derived growth factors required for their proliferation. Additionally, bisphosphonates were shown to exert direct antitumor action, as they inhibited tumor cell adhesion, invasion, and proliferation, and induced apoptosis of various human tumor cell lines in vitro [3]. However, to date this clear direct antitumor potential was not verified in vivo [10], due to the high affinity of bisphosphonates for bone mineral which must obstruct their availability for tumor cells. We undeniably observed a significantly higher potency of soluble compared to mineral-bound bisphosphonates at inhibiting tumor cell adhesion to bone in vitro [1]. Therefore strategies are needed to optimize bisphosphonates bioavailability and direct antitumor activity in vivo.

As well, cells are constantly changing their mechanical environment [20] and nanomechanical heterogeneity detection could facilitate damage detection in the extracellular matrix and improved remodelling responses. The heterogeneous nanomechanical prototypes measured experimentally could in turn induce local heterogeneous strains when loaded macroscopically. Such strains are expected to be increased by the softer surrounding cellular matrix of osteocytes [21] and also expected to influence interstitial fluid flow.

The objective of this work was to analyze the nanomechanical distribution of breast cancer bone metastasis before and after treatment of metastatic animals with Risedronate, the usually administrated drug in clinic. In this study, we have first analyzed by radiography, histology and histomorphometry the legs from metastatic mice and compared them with those from animals that had not been injected with breast cancer cells (naive mice).

By radiography, we have analyzed and compared the healthy bone without metastasis (Figure 2(a)), bone with metastasis induced after inoculation (Figure 2(b)) and the treated bone after treatment by Risedronate (Figure 2(c)).

After radiography on day 32 after tumor cell injection, we have shown that metastatic animals had large osteolytic lesions in hind limbs when compared to the bones of naive mice (Figure 2, panels b vs a). Metastatic animals were then treated either with the bisphosphonate Risedronate (administered by subcutaneous injection in

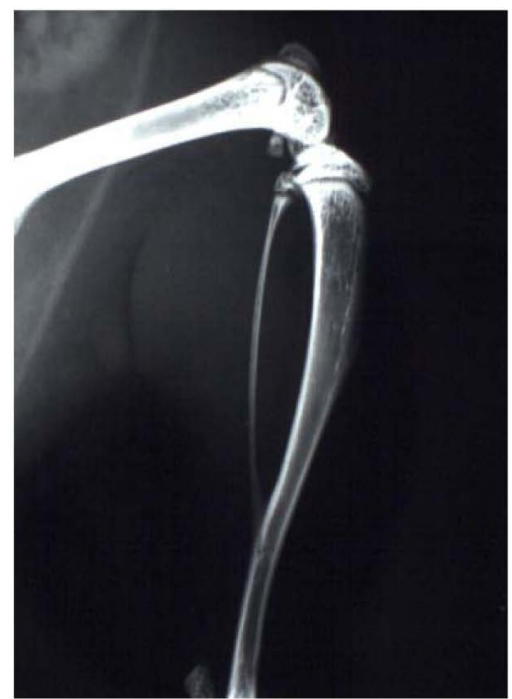

(a)

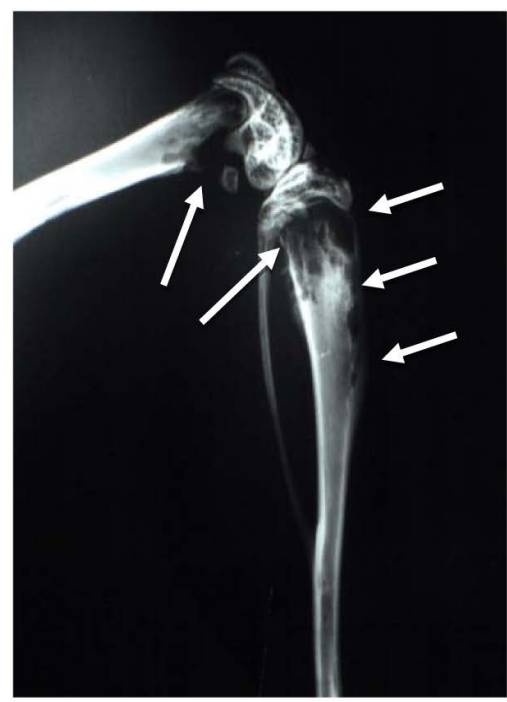

(b)

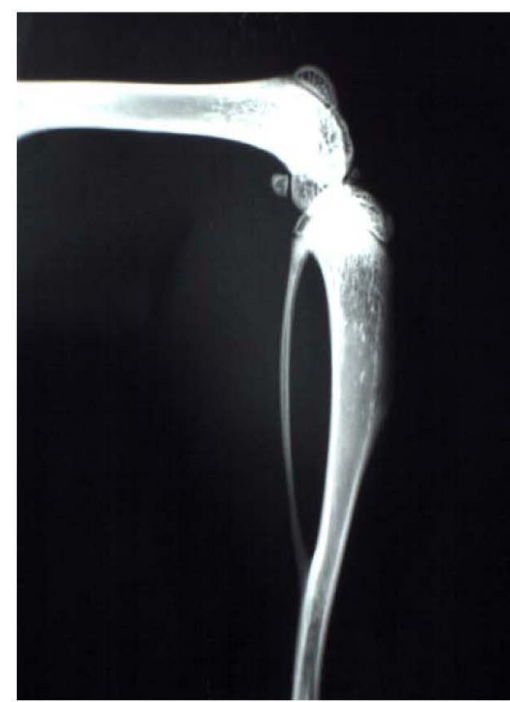

(c)

Figure 2. Identification of bone metastasis on radiographs of hind limbs from mice not injected with tumor cells with healthy bone, Naïve (a); b and c radiographs were obtained from different mice at day 32 after CHO- $\beta 3$ tumor cells inoculation. PBS was used as vehicle and corresponds to bone with metastasis (b); Risedronate was used to treat mice and the radiographs represent the treated bone (c). The radiographs displayed are examples that best illustrate the effects of treatments. Arrows indicate osteolytic lesions. 
PBS, used here as the vehicle) or the vehicle only.

After radiographic analysis, we examined the effect of Risedronate on the extent of bone destruction and determined the size of bone metastasis (Table 1 ).

Our results indicate clearly the antitumor efficacy of the treatment by Risedronate $\left(0.6 \mathrm{~mm}^{2}\right.$ for treated bone, compared to $5 \mathrm{~mm}^{2}$ for bone without treatment). We also performed histology and compared tumor burden and soft tissue volume ratio before and after treatment by Risedronate (Figure 3 and Table 2).

The images shown in Figure 3 are examples that best illustrate the effects of the treatments with Risedronate (Figure 3(c)) compared to no treated bone with tumor cells (asterisk in Figure 3(b)). To get more information
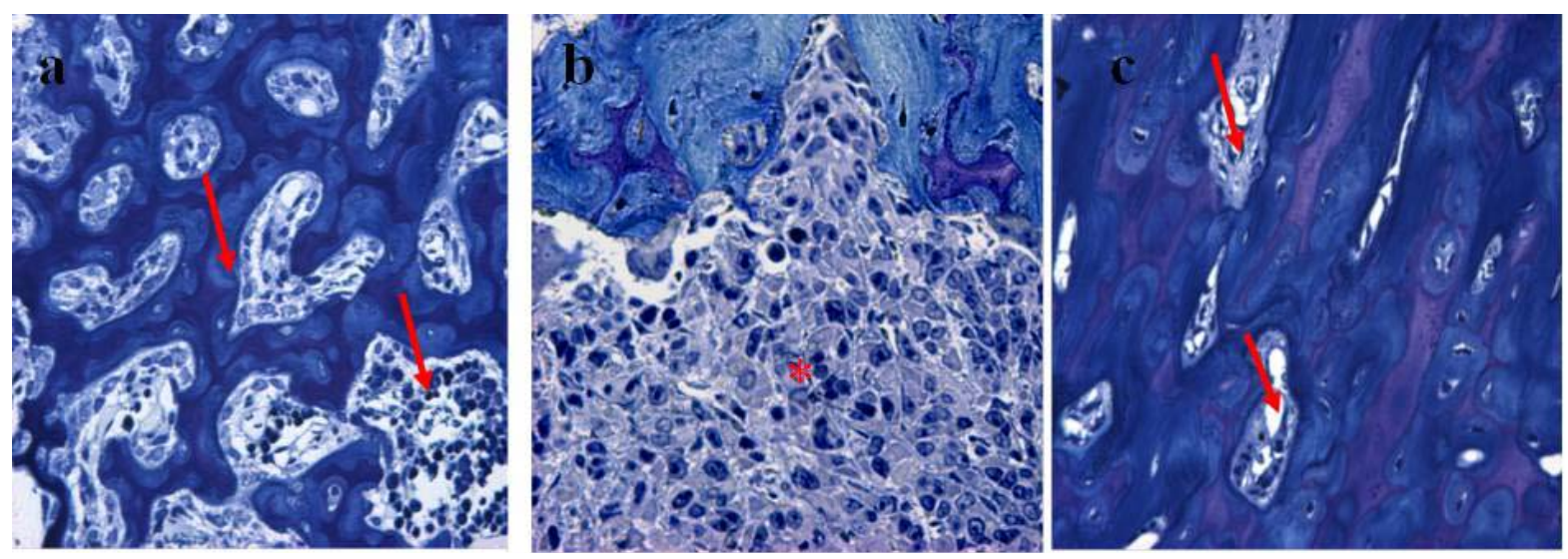

Figure 3. Optical microscopy visualization after semi-thin section $(7 \mu \mathrm{m})$ Histologic analysis of hind limbs from mice naïve with healthy bone (a); b and c were obtained from different mice at day 32 after CHO- $\beta 3$ tumor cells inoculation. PBS was used as vehicle and corresponds to bone with metastasis (b); Risedronate was used to treat mice and represent the treated bone (c). After toluidine blue stain, bone is stained blue and tumor cells zone were represented by asterisk. The arrows indicate the bone marrow.

Table 1. Tumor size determination after radiography.

\begin{tabular}{cc}
\hline Treatment" & Radiography (Tumor size (mm²/mouse) \\
Naive "healthy bone" & 0 \\
Vehicle "bone metastasis" & $5 \pm 0.6$ \\
Risedronate "treated bone" & $0.6 \pm 0.08^{\S}$
\end{tabular}

*Drug administration was initiated from the time of tumor cell inoculation (day 0) to the end of the protocol (day 32). All measurements were made 32 days after tumor cell injection. Naive animals had not been injected with tumor cells and correspond to the healthy bone. PBS was used as vehicle for animals injected with tumor cells and correspond to bone metastasis. Risedronate animals treated by daily dose of $150 \mu \mathrm{g} / \mathrm{kg}$, s.c. (clinical dose for patient with bone metastasis). Results are expressed as the mean $\pm \mathrm{SD}$ ( 2 Naive mice, 3 Vehicle mice and 3 Risedronate mice). ${ }^{\text {\$ }} \mathrm{P}<0.01$, compared to the vehicle-treated group. Statistical pairwise comparisons were made using Mann-Whitney U test.

Table 2. Histomorphometry from histology analysis.

\begin{tabular}{ccc}
\hline Treatment & & Histomorphometry \\
Naive "healthy bone" & BV/TV (\%) & 0 \\
Vehicle "bone metastasis" & $22.7 \pm 1.8^{\S}$ & $42.4 \pm 3.6$ \\
Risedronate "treated bone" & $9.5 \pm 2.6$ & $33.2 \pm 2.4^{\S}$ \\
\hline
\end{tabular}

Naive animals are mice that had not been injected with tumor cells. Risedronate $(150 \mu \mathrm{g} / \mathrm{kg}$, daily) was administered to metastatic animals by subcutaneous injection in $0.1 \mathrm{~mL}$ PBS (vehicle). Control mice bearing metastatic lesions received a daily treatment with the vehicle only. ${ }^{*}$ Drug administration was initiated from the time of tumor cell inoculation (day 0) to the end of the protocol (day 32). ${ }^{\dot{\tau}}$ Histomorphometry was performed on legs from naïve and metastatic animals. BV/TV: bone volume/tissue volume ratio (a measurement of the bone volume). TB/STV: tumor burden/soft tissue volume ratio (a measurement of the tumor volume). Results are expressed as the mean \pm SD (2 Naive mice, 3 Vehicle mice and 3 Risedronate mice). ${ }^{\S} \mathrm{P}$ $<0.01$, compared to the vehicle-treated group. Statistical pairwise comparisons were made using Mann-Whitney U test. 
concerning the quantification of bone, we analyzed finely by histomorphometry the histologic data and determined the ratio bone volume/tissue volume and tumor burden/soft tissues volume (Table 2).

The beneficial effect of the treatment is demonstrated by the increase of the ratio BV/TV from $9.5 \%$ for bone without treatment to $33.2 \%$ for the treated bone. We have also shown that after treatment the ratio TB/STV was $6.4 \%$ in comparison to $42.4 \%$ without treatment.

Metastatic animals treated with Risedronate had osteolytic lesions that were $95 \%$ smaller than those of tumor-bearing animals treated with the vehicle only (Table 1 and Figure 2(c)). Histologic analysis of hind limbs with metastases from vehicle-treated mice showed that tumor cells completely filled the bone marrow cavity and bone trabeculae were almost completely destroyed when compared to the bone histology of legs from naive mice (Figure 2, panels b vs a). By contrast, the extent of bone trabeculae in legs from Risedronate-treated mice was markedly increased, indicating a complete prevention of bone loss by the bisphosphonate (Figure 2(c)). In this respect, histomorphometric analysis of metastatic hind limbs from mice treated with Risedronate showed that the bone volume (BV) to tissue volume (TV) ratio was statistically significantly higher than that corresponding to vehicle-treated animals and naive mice (Table 2 and Figure 2). Risedronate also decreased the tumor burden (TB) to soft tissue volume (STV) ratio by 85\% compared with vehicle (Table 2 and Figure 2).

In order to get more information about the structure of bone after Risedronate treatment, we analyzed and compared by electron microscopy the bones from naive animals with those obtained from metastatic animals treated with the bisphosphonate. By optical microscopy, we visualized different cells (Figure 3) after semi-thin sections $(7 \mu \mathrm{m})$ of the different bone and toluidine blue stain. Figure 3(b) shows the invasion of bone by tumor cells working harder to destroy bone. In Figure 3(a) and Figure 3(c), we have a normal bone with bone marrow section and mineralized bone with osteoblasts and osteoclasts. In Figure 3(c), we can also see more mineralization than in Figure 3(a). This result could be explained by the capacity of Risedronate to induce mineralization.

Bones from naive animals and metastatic animals treated or not with Risedronate were next analyzed by Atomic Force Microscopy (AFM) in order to examine the ultrastructure and nanomechanical spatial heterogeneity of the bone tissue (Figure 4).

The raw indentation curves were obtained by atomic force microscopy with diamond-like tip (Figure 4(a)). We report here the first demonstration of the ultrastructure and nanomechanical spatial heterogeneity of bone

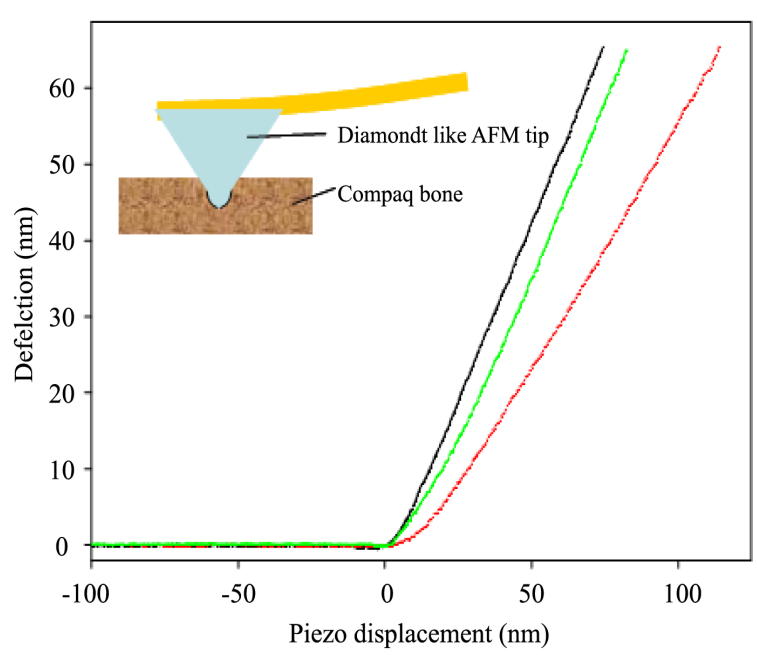

(a)

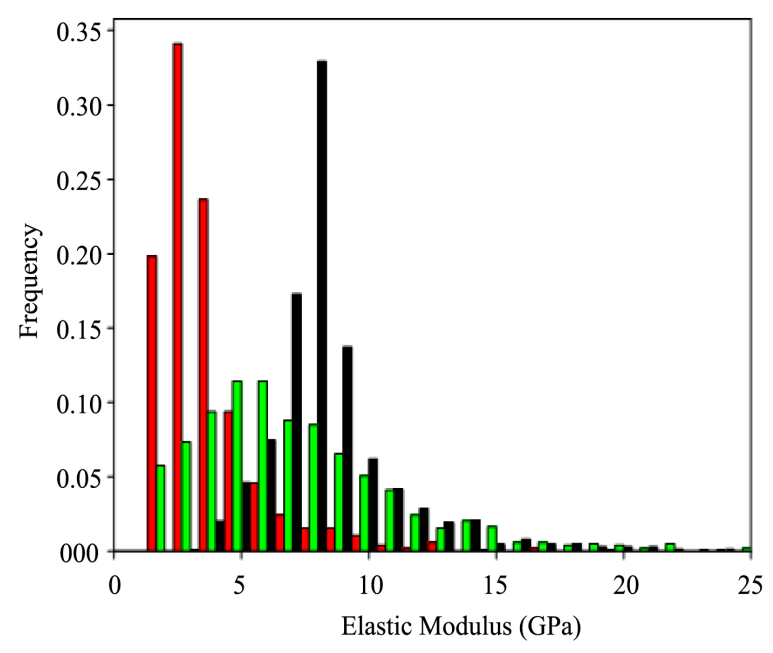

(b)

Figure 4. Representative raw indentation curves (a) and comparison of bone stiffness distribution (b). (a) Raw indentation curves obtained by atomic force microscopy with diamond like tip (100 nm of radius) for naïve healthy bone (black bar), vehicle bone with metastasis (red bar) and Risedronate treated bone (green bar) selected in the highest value of distribution. (b) Bone stiffness distributions for naive healthy bone (black bars) with a median of $8.14 \mathrm{GPa}$ (first quartile $=6.39 \mathrm{GPa}$, third quartile $=10.33 \mathrm{GPa}$ ), for bone with metastasis and Risedronate treated bone (green bars) with a median of $5.45 \mathrm{GPa}$ (first quartile $=3.49 \mathrm{GPa}$, third quartile $=8.08 \mathrm{GPa}$ ), and vehicle bone with metastasis (red bars) with a median of $2.38 \mathrm{GPa}$ (first quartile $=1.60 \mathrm{GPa}$, third quartile $=3.39 \mathrm{GPa}$ ), obtained after computation of the elastic modulus from nanoindentation data. 
stiffness with metastasis and the effect of the treatment on this nanomechanical distribution. The distribution of elastic modulus highlights the major decrease of elastic modulus in bone with metastasis (see histograms above). The median value is divided by a factor larger than 3 (8.14 GPa for healthy bone, $2.38 \mathrm{GPa}$ for bone with metastasis). The Risedronate treatment reduces this decrease with an intermediate median value of $5.45 \mathrm{GPa}$. The differences in median and quartile interval underscore in particular the strong softening of malignant bone with respect to the healthy bone. Furthermore, it should be emphasized that the Risedronate treatment leads to a significant recovering of the nanomechanical stiffness. To observe more finely these differences, spatial distribution of elastic modulus has been recorded (Figure 5).

In Figure 5, for each nanoindentation data spaced of $300 \mathrm{~nm}$, the elastic modulus is represented by a colour value. The three types of bone present heterogeneity in the spatial elastic modulus repartition (Figure 5(b),

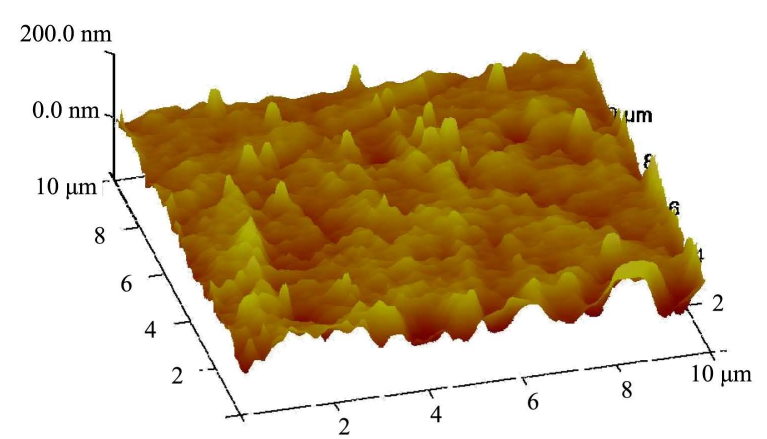

(a)

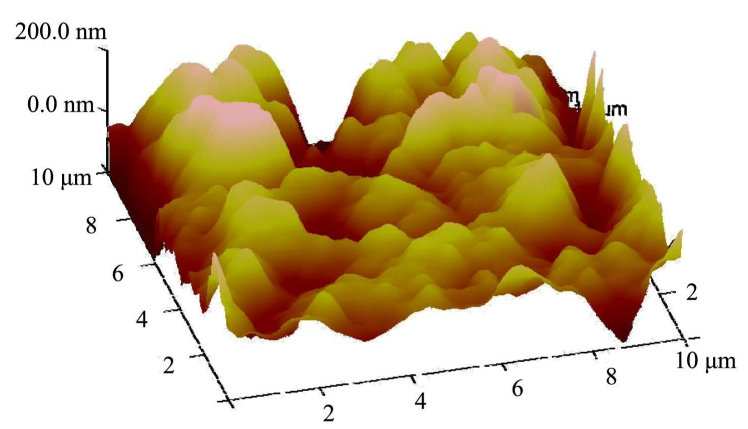

(c)

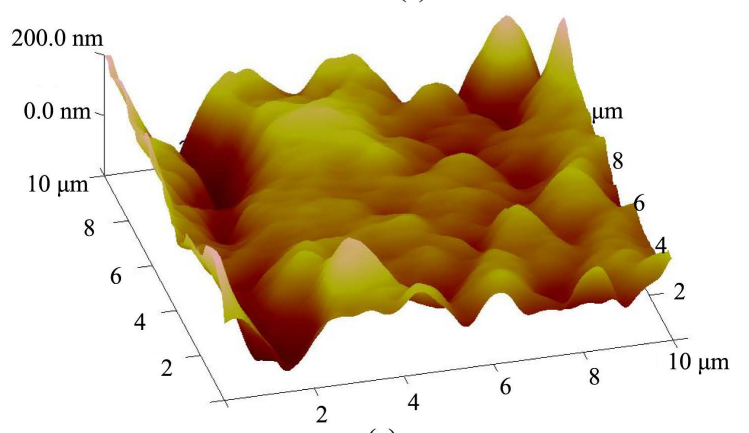

(b)

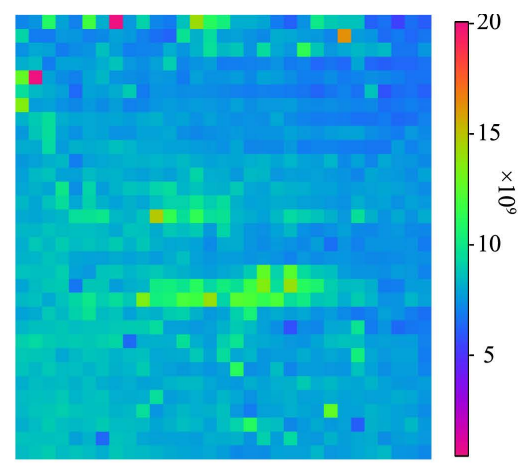

(d)

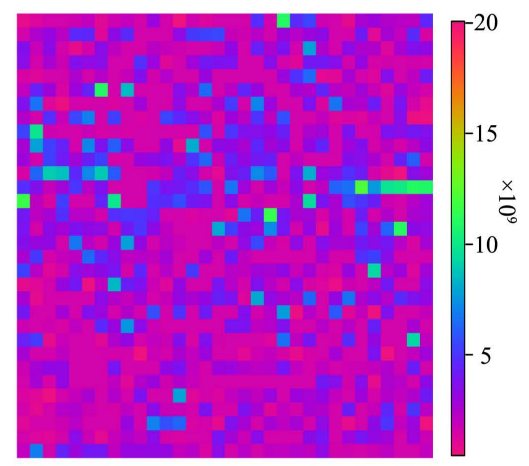

(f)

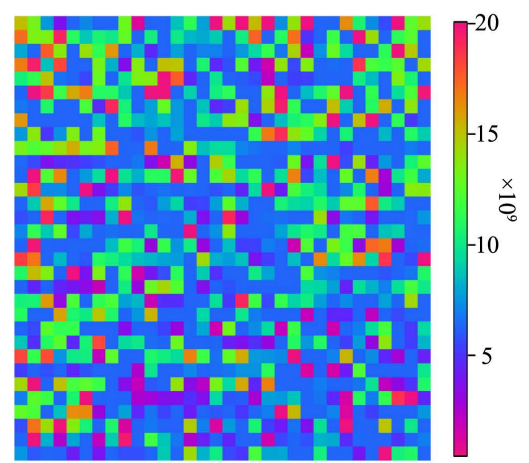

(e)

Figure 5. Ultrastructure and nanomechanical spatial heterogeneity of bone stiffness. (a) An a.c. intermittent contact-mode AFM height image $\left(10 \times 10 \mu^{2}\right)$ viewed perpendicular to the naive healthy bone axis with a RMS of $21.0 \mathrm{~nm}$; (b) 2D contour map $\left(10 \times 10 \mu^{2}\right)$ of elastic modulus calculated from nanoindentation data (c) An a.c. intermittent contact-mode AFM height image $\left(10 \times 10 \mu^{2}\right)$ viewed perpendicular to the vehicle bone with metastasis axis with a RMS of $55.3 \mathrm{~nm}$. (d) 2D contour map $\left(10 \times 10 \mu^{2}\right)$ of elastic modulus calculated from nanoindentation data; (e) An a.c. intermittent contact-mode AFM height image $\left(10 \times 10 \mu^{2}\right)$ viewed perpendicular to the Risedronate treated bone axis with a RMS of $50.1 \mathrm{~nm}$; (f) 2D contour map $\left(10 \times 10 \mu \mathrm{m}^{2}\right)$ of elastic modulus calculated from nanoindentation data. 
Figure 5(d), Figure 5(f)), but the bone with metastasis and Risedronate treatment is particular heterogeneous as revealed by the wide range of values of $\mathrm{E}$ present in the $10 \times 10 \mu \mathrm{m}^{2}$ sample (Figure $5(\mathrm{f})$ ).

In summary, bone is constantly being broken down and renewed. It is living tissue that needs exercise to gain strength. Bone metastasis occurs when cancer cells from the primary tumor relocate to the bone. Metastatic bone disease develops as a result of the many interactions between tumor cells and bone cells. This leads to disturbance of normal bone metabolism, with the increased osteoclast activity seen in most, if not all, tumor types providing a rational target for treatment.

Like many natural materials, bone is mechanically heterogeneous with spatial distributions in the shape, size and composition of its constituent. It is expected that nanomechanical heterogeneity influence elasticity, damage, fracture and remodelling of bone. We report here the first demonstration of the nanoscale stiffness distribution in bone metastasis before and after treatment of animals with Risedronate, a drug which is currently used for the treatment of bone metastases in patients with advanced cancers. This concept is generally applicable to a broad class of natural materials because nanomechanical heterogeneity expected to be ubiquitously presented.

\section{Methods}

\subsection{Specific Drug for Treatment}

Bisphosphonate Risedronate [2-(3-pyridinyl)1-hydroxyethylidene-bisphosphonic acid] was obtained from Procter and Gamble Pharmaceuticals (Mason, OH, USA). The drug was dissolved in water and stored at $4^{\circ} \mathrm{C}$.

\subsection{Mouse Model of Breast Cancer Bone Metastasis}

All procedures involving mice including their housing and care, the method by which they were killed, and all experimental protocols were conducted in accordance with a code of practice established by the ethical committee in Lyon (France). This study was monitored on a routine basis by the attending veterinarian according to ensure continued compliance with the proposed protocols. Four-week-old female Balb/c athymic (nu/nu) mice were purchased from Charles River (St. Germain sur l'Arbresle, France). The bone metastasis experiments in mice were conducted as previously described, using B02 cells, a subpopulation of the human MDA-MB-231 breast cancer cell line that was selected for the high efficiency with which it metastasizes to bone after intravenous inoculation [22]. B02 cells $(5 \times 105$ cells in $100 \mu \mathrm{L}$ phosphate-buffered saline) were injected into the tail vein of anesthetized (130 mg/kg ketamin and $8.8 \mathrm{mg} / \mathrm{kg}$ xylazin) mice on day 0 . Based on an average body weight of $20 \mathrm{~g}$ for 4 -wk-old mice, risedronate $(150 \mu \mathrm{g} / \mathrm{kg}$ body weight) was given daily to animals by subcutaneous injection in $100 \mu \mathrm{L}$ PBS (vehicle). Control mice received a daily treatment with the vehicle only. On day 32 after tumor cell inoculation, radiographs of anesthesized animals were taken with the use of MIN-R2000 film (Kodak) in an MX-20 cabinet X-ray system (Faxitron X-ray Corporation). Osteolytic lesions are recognized on radiographs as demarcated radiolucent lesions in the bone. The area of the osteolytic lesions was measured using a Visiolab 2000 computerized image analysis system (Explora Nova, La Rochelle, France) and the extent of bone destruction per animal was expressed in $\mathrm{mm}^{2}$, as described previously [22]. Anesthetized animals were killed by cervical dislocation following radiography at day 32.

\subsection{Bone Histology and Histomorphometry}

Bone histology and histomorphometric analysis of bone tissue sections were performed as previously described [22]. Vehicle- and bisphosphonate-treated tumor-bearing animals were killed at day 32, and both hind limbs from each animal were dissected, fixed in $80 \%$ (vol/vol) alcohol, dehydrated, and embedded in methylmethacrylate. A microtome (Polycut E, Reichert-Jung, Heidelberg, Germany) was used to cut $7 \mu \mathrm{m}$ thick sections of undecalcified long bones, and the sections were stained with Goldner's trichrome. Histologic and histomorphometric analyses were performed on Goldner-stained longitudinal medial sections of tibial metaphysis with the use of a computerized image analysis system (Visiolab 2000). Histomorphometric measurements [bone volume (BV)/tissue volume (TV) and tumor volume (TV)/soft tissue volume (STV) ratios] were performed in a standard zone of the tibial metaphysis, situated at $0.5 \mathrm{~mm}$ from the growth plate, including cortical and trabecular bone. The BV/TV ratio represents the percentage of bone tissue. The TV/STV ratio represents the percentage of tumor tissue. 


\subsection{Sample Preparation and Characterization}

\subsubsection{Sample Preparation}

Condylar tibia metaphysis were carefully sectioned under PBS (Gibco) flow by means of a sawing machine equipped with a diamond disk (Isomet, Buehler, Evanston, IL) into transversal sections about $2 \mathrm{~mm}$ thick from the proximal top (200 rpm speed).

Distal surfaces of sections were first set on AFM disks (Ted Pella Inc., Redding, CA) with wax (Kerr) and polished under distilled water flow by means of gradually disks fixed on polishing machine (Escil, Chassieu, France): G 1200, G 4000 silicon carbide and $3 \mu \mathrm{m}, 0.1 \mu \mathrm{m}$ microabrasive diamond polyester disks (40 rpm speed). Samples were stored in PBS solution until imaging and measuring.

The Tapping Mode ${ }^{\mathrm{TM}}$ AFM imaging at room humidity and temperature was used with Multimode AFM (Veeco, Santa Barbara, CA) on hydrated samples with a silicon probe MPP11100 (Veeco, Santa Barbara, CA) with tip radius about $10 \mathrm{~nm}$ and a resonant constant of $230 \mathrm{kHz}$. The roughness was determined by Root Mean Squared (RMS) approach on a $10 \times 10 \mu^{2}$ AFM images.

\subsubsection{Nanoindentation}

Nanoindentation experiments were analyzed in ambient conditions using the PicoForce microscope (Veeco, Santa Barbara, CA) and NW-DT-NCHR cantilevers $\left(42 \mathrm{~N} \cdot \mathrm{m}^{-1}\right.$ of spring stiffness, $100 \mathrm{~nm}$ of tip radius, Nano World, Schaffhausen, Switzerland). Stiffness (dynamic elastic modulus, E) measurements of bone samples were performed as described previously [18]. Tai et al. verified that loading/unloading rates between $0.5-5 \mu \mathrm{m} \cdot \mathrm{s}^{-1}$ did not lead to statistically representative differences in calculated bone moduli [18]. Therefore, we carried out displacement-controlled nanoindentation by loading at a rate of $1 \mu \mathrm{m} \cdot \mathrm{s}^{-1}$ up to a trigger force of $5 \mu \mathrm{N}$ followed by unloading at the same rate. To derive the elastic modulus, $E$, we use the Hertzian relation, derived for paraboloidal tips, between applied force, $F$, and resulting indentation, $\delta$, namely

$$
F=\frac{4}{3} \frac{E}{1-v^{2}} R^{1 / 2} \delta^{3 / 2}
$$

where $v$ is the Poisson ratio and $R$ is the tip radius. The Poisson ratio for cortical bone is 0.325 [23]. Values of the elastic modulus were derived from the experimental force vs. indentation curve over the indentation domain ranging from $20 \%$ up to $95 \%$ of the maximum indentation corresponding to the maximum applied force of $5 \mu \mathrm{N}$. A minimum of $100 \mathrm{~nm}$ inter-indentation spacing was considered to be sufficiently large for minimal interference with adjacent residual in elastically deformed zone as well as remaining stresses.

\subsection{Statistical Analysis}

The three histograms shown in Figure 4(b) have been compared using One-Way ANOVA on ranks (i.e. Kruskal-Wallis test; Sigmastat, Systat Software, Chicago, IL). They differ significantly $(\mathrm{P}<0.001)$. Consequently, a pairwise comparison was undertaken (using Dunn's method) which showed that the three pairs of groups differed significantly $(\mathrm{P}<0.05)$.

\subsection{Histological and Electron Microscopic Analysis}

The samples were fixed in Karnovsky fixative, postfixed with $1 \%$ osmium tetroxide in $0.1 \mathrm{M}$ cacodylate buffer for $1 \mathrm{~h}$ at $4^{\circ} \mathrm{C}$, dehydrated through graded alcohol and embedded in Epon 812. Semi-thin sections were cut at 7 $\mu \mathrm{m}$ and stained with toluidine blue, and histologically analysed by light microscopy. Ultrathin sections were cut at $70 \mathrm{~nm}$ and contrasted with uranyl acetate and lead citrate, and examined with a Morgagni 268 electron microscope.

\section{Acknowledgements}

This work was supported by the NanoOSCAR ANR project from the "Agence Natiionale la Recherche", the "Fondation Avenir", the "Ligue contre le Cancer du Haut-Rhin, Région Alsace" and "Cancéropôle du Grand Est". LK and QW thank the "Faculté de Chirurgie Dentaire" of Strasbourg. Histology was made by Dr N. Messadeq. 


\section{References}

[1] Boissier, S., Magnetto, S., Frappart, L., Cuzin, B., Ebetino, F.H., Delmas, P.D. and Clezardin, P. (1997) Bisphosphonates Inhibit Prostate and Breast Carcinoma Cell Adhesion to Unmineralized and Mineralized Bone Extracellular Matrices. Cancer Research, 57, 3890-3894.

[2] Coleman, R.E. (2008) Risks and Benefits of Bisphosphonates. British Journal of Cancer, 98, 1736-1740. http://dx.doi.org/10.1038/sj.bjc.6604382

[3] Stresing, V., Daubine, F., Benzaid, I., Monkkonen, H. and Clezardin, P. (2007) Bisphosphonates in Cancer Therapy. Cancer Letters, 257, 16-35. http://dx.doi.org/10.1016/j.canlet.2007.07.007

[4] Fantner, G.E., Hassenkam, T., Kindt, J.H., Weaver, J.C., Birkedal, H., Pechenik, L., Cutroni, J.A., Cidade, G.A., Stucky, G.D., Morse, D.E. and Hansma, P.K. (2005) Sacrificial Bonds and Hidden Length Dissipate Energy as Mineralized Fibrils Separate during Bone Fracture. Nature Materials, 4, 612-616. http://dx.doi.org/10.1038/nmat1428

[5] Gao, H., Ji, B., Jager, I.L., Arzt, E. and Fratzl, P. (2003) Materials Become Insensitive to Flaws at Nanoscale: Lessons from Nature. Proceedings of the National Academy of Sciences of the United States of America, 100, 5597-5600. http://dx.doi.org/10.1073/pnas.0631609100

[6] Gupta, H.S., Wagermaier, W., Zickler, G.A., Raz-Ben Aroush, D., Funari, S.S., Roschger, P., Wagner, H.D. and Fratzl, P. (2005) Nanoscale Deformation Mechanisms in Bone. Nano Letters, 5, 2108-2111. http://dx.doi.org/10.1021/nl051584b

[7] Tai, K., Ulm, F.J. and Ortiz, C. (2006) Nanogranular Origins of the Strength of Bone. Nano Letters, 6, 2520-2525. http://dx.doi.org/10.1021/nl061877k

[8] Morgan, E.F., Bayraktar, H.H. and Keaveny, T.M. (2003) Trabecular Bone Modulus-Density Relationships Depend on Anatomic Site. Journal of Biomechanics, 36, 897-904. http://dx.doi.org/10.1016/S0021-9290(03)00071-X

[9] Pope, M.H. and Outwater, J.O. (1974) Mechanical Properties of Bone as a Function of Position and Orientation. Journal of Biomechanics, 7, 61-66. http://dx.doi.org/10.1016/0021-9290(74)90070-0

[10] Gupta, H.S., Stachewicz, U., Wagermaier, W., Roschger, P., Wagner, H.D. and Fratzl, P. (2006) Mechanical Modulation at the Lamellar Level in Osteonal Bone. Journal of Materials Research, 21, 1913-1921. http://dx.doi.org/10.1557/jmr.2006.0234

[11] Rho, J.Y., Roy, M.E., 2nd, Tsui, T.Y. and Pharr, G.M. (1999) Elastic Properties of Microstructural Components of Human Bone Tissue as Measured by Nanoindentation. Journal of Biomedical Materials Research, 45, 48-54. http://dx.doi.org/10.1002/(SICI)1097-4636(199904)45:1<48::AID-JBM7>3.0.CO;2-5

[12] Martin, R.B. and Burr, D.B. (1989) Structure, Function and Adaptation of Compact Bone. Raven Press, New York.

[13] Balooch, G., Balooch, M., Nalla, R.K., Schilling, S., Filvaroff, E.H., Marshall, G.W., Marshall, S.J., Ritchie, R.O., Derynck, R. and Alliston, T. (2005) TGF-Beta Regulates the Mechanical Properties and Composition of Bone Matrix. Proceedings of the National Academy of Sciences of the United States of America, 102, 18813-18818. http://dx.doi.org/10.1073/pnas.0507417102

[14] Jaasma, M.J., Bayraktar, H.H., Niebur, G.L. and Keaveny, T.M. (2002) Biomechanical Effects of Intraspecimen Variations in Tissue Modulus for Trabecular Bone. Journal of Biomechanics, 35, 237-246. http://dx.doi.org/10.1016/S0021-9290(01)00193-2

[15] Peterlik, H., Roschger, P., Klaushofer, K. and Fratzl, P. (2006) From Brittle to Ductile Fracture of Bone. Nature Materials, 5, 52-55. http://dx.doi.org/10.1016/S0021-9290(01)00193-2

[16] Phelps, J.B., Hubbard, G.B., Wang, X. and Agrawal, C.M. (2000) Microstructural Heterogeneity and the Fracture Toughness of Bone. Journal of Biomedical Materials Research, 51, 735-741. http://dx.doi.org/10.1002/1097-4636(20000915)51:4<735::AID-JBM23>3.0.CO;2-G

[17] Currey, J. (2005) Structural Heterogeneity in Bone: Good or Bad? Journal of Musculoskeletal \& Neuronal Interactions, $5,317$.

[18] Tai, K., Dao, M., Suresh, S., Palazoglu, A. and Ortiz, C. (2007) Nanoscale Heterogeneity Promotes Energy Dissipation in Bone. Nature Materials, 6, 454-462. http://dx.doi.org/10.1038/nmat1911

[19] Engler, A.J., Richert, L., Wong, J.Y., Picart, C. and Discher, D.E. (2004) Surface Probe Measurements of the Elasticity of Sectioned Tissue, Thin Gels and Polyelectrolyte Multilayer Films: Correlations between Substrate Stiffness and Cell Adhesion. Surface Science, 570, 142-154. http://dx.doi.org/10.1016/j.susc.2004.06.179

[20] Ehrlich, P.J. and Lanyon, L.E. (2002) Mechanical Strain and Bone Cell Function: A Review. Osteoporosis International, 13, 688-700. http://dx.doi.org/10.1007/s001980200095

[21] You, L., Cowin, S.C., Schaffler, M.B. and Weinbaum, S. (2001) A Model for Strain Amplification in the Actin Cy- 
toskeleton of Osteocytes Due to Fluid Drag on Pericellular Matrix. Journal of Biomechanics, 34, 1375-1386. http://dx.doi.org/10.1016/S0021-9290(01)00107-5

[22] Peyruchaud, O., Serre, C.M., NicAmhlaoibh, R., Fournier, P. and Clezardin, P. (2003) Angiostatin Inhibits Bone Metastasis Formation in Nude Mice through a Direct Anti-Osteoclastic Activity. Journal of Biomechanics, 278, 45826-45832

[23] Cowin, S.C. (1999) Bone Poroelasticity. Journal of Biomechanics, 32, 217-238.

http://dx.doi.org/10.1016/S0021-9290(98)00161-4 